\title{
Toward reduction of artifacts in fused images
}

\author{
Mario Lillo-Saavedra ${ }^{\mathrm{a}, *}$, Consuelo Gonzalo ${ }^{\mathrm{b}}$, Octavio Lagos ${ }^{\mathrm{a}}$ \\ ${ }^{a}$ Facultad de Ingeniería Agrícola, Universidad de Concepción, Av. Vicente Mendez 597, casilla 537, Chillán, Chile \\ ${ }^{\mathrm{b}}$ Facultad de Informática, Universidad Politécnica de Madrid, Campus Montegancedo, Boadilla del Monte, 28660 Madrid, Spain
}

Keywords:

Fractal dimension

Fusion image

Wavelet transform

Texture

\begin{abstract}
A B S T R A C T
Most fusion satellite image methodologies at pixel-level introduce false spatial details, i.e. artifacts, in the resulting fused images. In many cases, these artifacts appears because image fusion methods do not consider the differences in roughness or textural characteristics between different land covers. They only consider the digital values associated with single pixels. This effect increases as the spatial resolution image increases. To minimize this problem, we propose a new paradigm based on local measurements of the fractal dimension (FD). Fractal dimension maps (FDMs) are generated for each of the source images (panchromatic and each band of the multi-spectral images) with the box-counting algorithm and by applying a windowing process. The average of source image FDMs, previously indexed between 0 and 1 , has been used for discrimination of different land covers present in satellite images. This paradigm has been applied through the fusion methodology based on the discrete wavelet transform (DWT), using the à trous algorithm (WAT). Two different scenes registered by optical sensors on board FORMOSAT-2 and IKONOS satellites were used to study the behaviour of the proposed methodology. The implementation of this approach, using the WAT method, allows adapting the fusion process to the roughness and shape of the regions present in the image to be fused. This improves the quality of the fused images and their classification results when compared with the original WAT method.
\end{abstract}

\section{Introduction}

Recent improvements in technologies of Earth observation from space have resulted in a wide range of commercial satellites that offer a new generation of images. Among them, FORMOSAT-2 with a spatial resolution of $2 \mathrm{~m}$ in the panchromatic and $8 \mathrm{~m}$ in multispectral bands; IKONOS and KOMPSAT-2 with $1 \mathrm{~m}$ (panchromatic) and $4 \mathrm{~m}$ (multispectral bands) respectively; QUICKBIRD with $0.61 \mathrm{~m}$ and $2.44 \mathrm{~m}$; and GEOEYE- 1 with $0.41 \mathrm{~m}$ and $1.65 \mathrm{~m}$, can be mentioned. Additionally, third generation satellites will be capable of discriminating objects on the Earth's surface with sizes close to $0.25 \mathrm{~m}$ (GEOEYE-2). Considering the existence of a wide range of spatial resolutions, it is possible to establish that images with a spatial resolution of less than $1 \mathrm{~m}$ are considered very high resolution images, as is the case for most of the panchromatic images mentioned before, and images with spatial resolution ranging from $4 \mathrm{~m}$ to $1 \mathrm{~m}$ are considered high resolution images.

Even when an increase in spatial resolution simplifies the problem of mixing pixels, other problems emerge due to the greater spatial variability for each spectral class present in the image; furthermore, the limited number of spectral bands of high and very

\footnotetext{
* Corresponding author. Tel.: +56 42208807; fax: +56 42275303.

E-mail address: malillo@udec.cl (M. Lillo-Saavedra).
}

high spatial resolution images produces low separability between land covers (Bruzzone and Carlin, 2006; Huang et al., 2008).

Today, a plethora of image fusion algorithms is available (Mitchell, 2010), most of them based on the use of different types of transforms. Some are very simple from a conceptual point of view, such as methods based on Brovey transform (Hallada and Cox, 1983), ог IHS transform (Intensity, Hue, Saturation) (Chavez et al., 1991). These type of methodologies have been shown to provide fused images with considerable colour distortions when compared to the colour of the original multi-spectral image (Choi, 2006; Tu et al., 2001).

Fusion methods based on multi-resolution analysis techniques produce fused images with higher colour quality. Most of these techniques use the Discrete Wavelet Transform (DWT), both in its pyramidal and redundant versions (Gonzalez-Audicana et al., 2005; Gonzalo and Lillo-Saavedra, 2008; Li et al., 2010; Mallat, 1989; Pohl and Van Genderen, 1998; Zhou et al., 1998). Since the appearance of new transforms, such as Curvelets (Candes and Donoho, 2000; Do and Vetterli, 2001), Ridgelets (Candes and Donoho, 1999) and Contourlets (Do and Vetterli, 2005), new fusion methodologies based on these approaches have been proposed (Lillo-Saavedra and Gonzalo, 2007; Nencini et al., 2007; Qu et al., 2008; Yang et al., 2009; Zhang and Guo, 2009). Even though these methods increase the spatial resolution of multi-spectral images to the spatial resolution levels of panchromatic images, maintaining their spectral 
characteristics, they tend to accentuate even more the problem of injecting false details into the fused images (artifacts) (Gonzalo et al., 2008; Toet et al., 2010; Zribi, 2010). Thus, new strategies to process these kinds of satellite images are required.

Different approaches have been identified in the literature to handle this problem: some are based on the use of new classification techniques that are especially adapted to these types of images (Alonso et al., 2007; Bruzzone and Carlin, 2006; Huiping et al., 2003 ) and others are oriented to image processing. Grazzini et al. (2005) suggests a solution of smoothing heterogeneous areas while preserving the main edges of the images by using a multi-fractal reconstruction method.

These aspects have not been thoroughly investigated with fusion image techniques. However, a few works are found in the literature. Huang et al. (2008) offer a fusion method based on the extraction of characteristics at different scales, using the DWT, in order to improve the classification results of the fused images. Gonzalo et al. (2008) have proposed an improved version of the generalized IHS fusion algorithm (Tu et al., 2001) considering the roughness of the land covers. Even though the generalized IHS algorithm (Choi, 2006; Tu et al., 2004) is quite limited regarding the spectral quality of fused images, the results produced have been auspicious.

The fusion methodology proposed by Gonzalo et al. (2008), preserves the high frequency information of the panchromatic image in areas with high detail, while in homogeneous areas the low frequency information from the multi-spectral image is conserved. Thus, if the area corresponds to homogeneous coverage, such as full cover crops or prairies, a small amount of spatial information should be incorporated from inside this area, maintaining a more homogeneous spectral response, closer to the original multispectral image. Moreover, if the coverage is not very homogeneous (i.e. urban areas and partial crop coverage), more spatial information, from the panchromatic image, should be integrated into the fused image.

Some studies (Arduini et al., 1991; Karathanassi et al., 2007; Li et al., 2008; Myint, 2003; Sun et al., 2006) have shown the ability of fractal geometry to describe irregular forms and complex objects present in natural surfaces, making it especially adequate to analyze these surfaces.

The majority of fractal geometry applications for image analysis are based on the calculation of the fractal dimension (FD). This indicator provides a measurement of irregularity for complex objects as well as the homogeneity of uniform surfaces. Rougher surfaces have a higher fractal dimension in comparison with more homogeneous surfaces (Pentland, 1984).

The present paper proposes a new image fusion paradigm based on local measurements of the FD. It will be applied to the Discrete Wavelet Transform fusion methodology, implemented through the à trous algorithm (WAT) (Lillo-Saavedra and Gonzalo, 2006). This paradigm will be called fractal dimension maps fusion (FDMF), where the fractal dimension maps (FDMs) are estimated by the box-counting algorithm (Conci and Nunes, 2001; Parrinello and Vaughan, 2002; Sun, 2006), using a windowing process (see Appendix A).

\section{Methods and materials}

\subsection{Weighted WAT fusion through FDMs}

As mentioned above, the fusion methodology proposed in this paper is based on the discrete wavelet transform (DWT) fusion methodology, calculated by the à trous algorithm (WAT) (LilloSaavedra and Gonzalo, 2006). Eq. (1) includes the definition of the ith band of the fused image $\left(I_{F U S}^{i}(x, y)\right)$ through the WAT method:

$I_{F U S}^{i}(x, y)=I_{M U L_{j}}^{i}(x, y)+\sum_{k=1}^{W} C_{P A N_{k}}(x, y)$

where the indices $i$ and $j$ represent the number of bands and the degradation level of the multi-spectral image, respectively; $W$ corresponds to the number of wavelet planes to be integrated from the panchromatic image into the last degradation level of the multispectral image ( $I_{M L_{j}}^{i}$ ); and $C_{\text {PAN }_{k}}$ corresponds to the panchromatic wavelet coefficients. This fusion strategy does not consider spectral differences between bands and cannot control the inherent tradeoff between spatial-spectral quality in the fused image (Choi, 2006; Garzelli et al., 2004; Lillo-Saavedra and Gonzalo, 2006).

Lillo-Saavedra and Gonzalo (2006) proposed a modification of the à trous algorithm, establishing a mechanism that controls this trade-off by introducing a wavelet coefficient weighting factor $\left(\alpha^{i}\right)$ for the panchromatic wavelet coefficients. The proposed fusion algorithm is formally represented in (2):

$I_{F U S}^{i}(x, y)=I_{M U L_{j}}^{i}(x, y)+\alpha^{i} \sum_{k=1}^{W} C_{P A N_{k}}(x, y)$

Despite the previously mentioned advantage of this fusion algorithm, the method is not capable of discriminating between different land covers present in images (Gonzalo et al., 2008). In that sense, the same value of $\alpha^{i}$ is used to weigh the information coming from the panchromatic wavelet coefficients for all spectral bands. This is the main cause of the artifacts in the fused images. To overcome this problem, in this work, $\alpha^{i}$ will be an array with same source image size, rather than a single number. Therefore, Eq. (2) can be rewritten as:

$I_{\text {FUS }}^{i}(x, y)=I_{M U L_{j}}^{i}(x, y)+\alpha_{\omega_{\text {sizes }}}^{i}(x, y) \sum_{k=1}^{W} C_{P_{A N}}(x, y)$

where $\alpha_{\omega_{\text {sizes }}}^{i}(x, y)$ has been estimated through the FDMs of the source images to be fused, that is each spectral band of the multispectral image and the panchromatic image. These FDMs are generated for each source image by a sliding window processing. Through this process, a FD value, associated with the window central pixel, is determined for each position. Obtaining, in this way, a FDM with the same size as the original image. It is important to note that even when a different weighting value is found for each pixel, this value depends on the pixel grey levels in its neighbourhood. Therefore, the window size, $\omega_{\text {sizes }}$, has an influence on the FDMs values (Gonzalo et al., 2008). Each element in these maps provides a different weighting value for each pixel and each band.

In this work, $\alpha_{\omega_{\text {sizes }}}^{i}(x, y)$ has been defined as the average value of both dimensions, previously indexed between 0 and 1 as formalized in (4). This approach increases the detail information in the fused image, avoiding the artifacts that occur when one considers only the FDM of the panchromatic image.

$\alpha_{\omega_{\text {sizes }}^{i}}^{i}(x, y)=\frac{\left\langle F D\left(I_{M U L}^{i}(x, y), \omega_{\text {sizes }}\right)\right\rangle+\left\langle F D\left(I_{\text {PAN }}(x, y), \omega_{\text {sizes }}\right)\right\rangle}{2}$

where $\langle\cdot\rangle$ represents the indexed fractal dimension values. These values are obtained by dividing the original fractal dimension values by the maximum value. This definition ensures that for rough coverages, characterized by a high fractal dimension value in at least one of source images, a large amount of spatial information will be integrated into the fused image. Whereas when the fractal dimension of coverage under analysis is low in both source images, the integration of spatial information will be low. 
Table 1

Fused images quality indices.

\begin{tabular}{|c|c|c|c|}
\hline Index & Equation & Ideal value & Reference \\
\hline$C C$ & $\frac{1}{N_{\text {Bards }}} \sum_{i=1}^{N_{\text {Bards }}} \operatorname{corr}_{i}\left(I_{M U L}^{i}, I_{F U S}^{i}\right)$ & 1 & Vijayaraj et al. (2004) \\
\hline ERGAS & $100 \frac{h}{l} \sqrt{\frac{1}{N P} \sum_{i=1}^{N_{B a n d s}}\left(\frac{R M S E\left(I_{M U L}^{i}, I_{\text {FUS }}^{i}\right)^{2}}{\left(f_{M U L}^{i}\right)^{2}}\right)}$ & 0 & Wald (2002) \\
\hline$Q$ & $\frac{4 \sigma_{I_{M U I I} I_{F U S}} \bar{I}_{M U L I} \bar{I}_{F U S}}{\left(\sigma_{I_{M U I}}^{2}+\sigma_{I_{F U S}^{2}}^{2}\right)\left[\left(\bar{I}_{M U L}\right)^{2}+\left(\bar{I}_{F U S}\right)^{2}\right]}$ & 1 & Wang and Bovik (2002) \\
\hline$S A M$ & $\sum_{b=1}^{N} i_{b}^{i_{b}} r_{b}$ & $0^{\circ}$ & Kruse et al. (1993) \\
\hline SSIM & $\frac{\left(2 \bar{I}_{F U S} \bar{I}_{M U L}+C 1\right)\left(2 \sigma_{I_{M U L}} I_{F U S}+C 2\right)}{\left(\bar{I}_{M U I}^{2}+\bar{I}_{F U S}^{2}+C 1\right)\left(\sigma_{I_{M U L}^{2}}^{2}+\sigma_{I_{F U S}^{2}}^{2}+C 2\right)}$ & 1 & Wang et al. (2004) \\
\hline$Z$ & $\frac{1}{N_{\text {Bards }}} \sum_{i=1}^{N_{B a n d s}} \operatorname{com}_{i}\left(I_{\text {PAN }}^{\text {high-pass }}, I_{\text {FuS }}^{I_{\text {high-puss }}}\right)$ & 1 & Zhou et al. (1998) \\
\hline
\end{tabular}

\subsubsection{Quality evaluation of fused images}

Different metrics have been proposed in the literature in order to measure the quality of fused images. There exists a wide range of statistical indices for that purpose (e.g. mean, correlation, covariance matrix, standard deviation). The two indices of this group most extensively used are: the spectral correlation (CC) (Vijayaraj et al., 2004), which estimates the spectral quality of the fused images, disregarding the spatial characteristics; and the spatial correlation (Z) (Zhou et al., 1998), which evaluates spatial quality without evaluating spectral characteristics. The main problem of using metrics based on correlations is the low sensitivity of these indices to quality variations (saturation effect). In other words, large differences in the fused image features do not correspond with very different values in the metrics.

Another extensively used quality index is the spectral angle mapping (SAM) (Kruse et al., 1993), which maps spectral similarity, using the $\cos ^{-1}$ of the dot products of image and reference images as $n$-dimensional vectors (where $n$ is the number of bands) and computes the spectral angle between them.

None of the metrics mentioned above can be used to simultaneously evaluate different features of the source images, such as the spatial and radiometric characteristics, and in this sense they are not considered to be global indices.

Global metrics have been proposed in several works (Alparone et al., 2004; Wald, 2002; Wang and Bovik, 2002). Wald (2002) was the first author to propose an index to measure the global quality of the products resulting from the image fusion process. This index was called Erreur Relative Globale Adimensionalle de Synthêse (ERGAS). Another global metric was proposed by Wang and Bovik (2002); it was called the Quality Universal Index (Q), which is based on different statistical properties of the fused and multispectral original images. All indices mentioned above are based on error sensitivity, which relies on a number of strong assumptions and generalizations not always completely true (Wang et al., 2004), such as a high correlation between image fidelity and image quality. Wang et al. (2004) proposed a new index (SSMI) based on structural similarity. They show that this new approach can correct some of sensitivity-based indices limitations. Morever, experimental results have shown that this index behaves in a similar way as global indices: ERGAS and $Q$. Table 1 summarizes the quality indices, sorted alphabetically, that have been used in this work, including the symbols, their equations, ideal values and references.

All quality indices included in Table 1 have a common feature: the fused image quality is evaluated by a unique value for the whole image (scalar index). However, due to the different coverages presented in an image, and their different responses to a particular fusion method, it is more convenient to measure the quality of the fused images using a map index rather than a scalar index. Therefore, in this paper, maps of the Q quality index have been calculated (Wang and Bovik, 2002). These quality maps have been obtained using a sliding window process with a window size of 11 . The values of $Q$ have been estimated in the neighbourhood of each pixel of the fused images.

\subsection{Data set}

In order to analyze the behaviour of the proposed method, two kinds of satellite images, with different spatial resolution and similar spectral resolution, were used: FORMOSAT-2 and IKONOS. Both images present the same relation $(1 / 4)$ between the spatial resolution of the panchromatic and the multi-spectral images. Table 2 presents their characteristics.

Fig. 1(a) and (b) shows colour compositions of the multi-spectral images, while Fig. 1(c) and (d) presents the respective panchromatic images for FORMOSAT-2 and IKONOS.

The FORMOSAT- 2 scene corresponds to an area of the University of Concepción, Chile (72 $\left.3^{\prime} 11^{\prime \prime} \mathrm{W}, 36^{\circ} 49^{\prime} 23^{\prime \prime} \mathrm{S}\right)$ recorded on April 28, 2008. Fundamentally, two coverage types appear in the image: urban and forest areas. The IKONOS scene presents a crop area located in the Maipo Valley, Metropolitan Region, Chile

Table 2

Spatial and spectral characteristics of the source images.

\begin{tabular}{|c|c|c|c|c|}
\hline \multirow[t]{2}{*}{ Band } & \multicolumn{2}{|l|}{ FORMOSAT-2 } & \multicolumn{2}{|l|}{ IKONOS } \\
\hline & Spatial resolution & Spectral resolution $(\mu \mathrm{m})$ & Spatial resolution & Spectral resolution $(\mu \mathrm{m})$ \\
\hline B1 & \multirow{4}{*}{$8 \mathrm{~m}$} & $0.450-0.520$ & \multirow{4}{*}{$4 \mathrm{~m}$} & $0.445-0.516$ \\
\hline B2 & & $0.520-0.600$ & & $0.506-0.595$ \\
\hline B3 & & $0.630-0.690$ & & $0.632-0.698$ \\
\hline B4 & & $0.760-0.900$ & & $0.757-0.853$ \\
\hline PAN & $2 \mathrm{~m}$ & $0.450-0.900$ & $1 \mathrm{~m}$ & $0.450-0.900$ \\
\hline
\end{tabular}



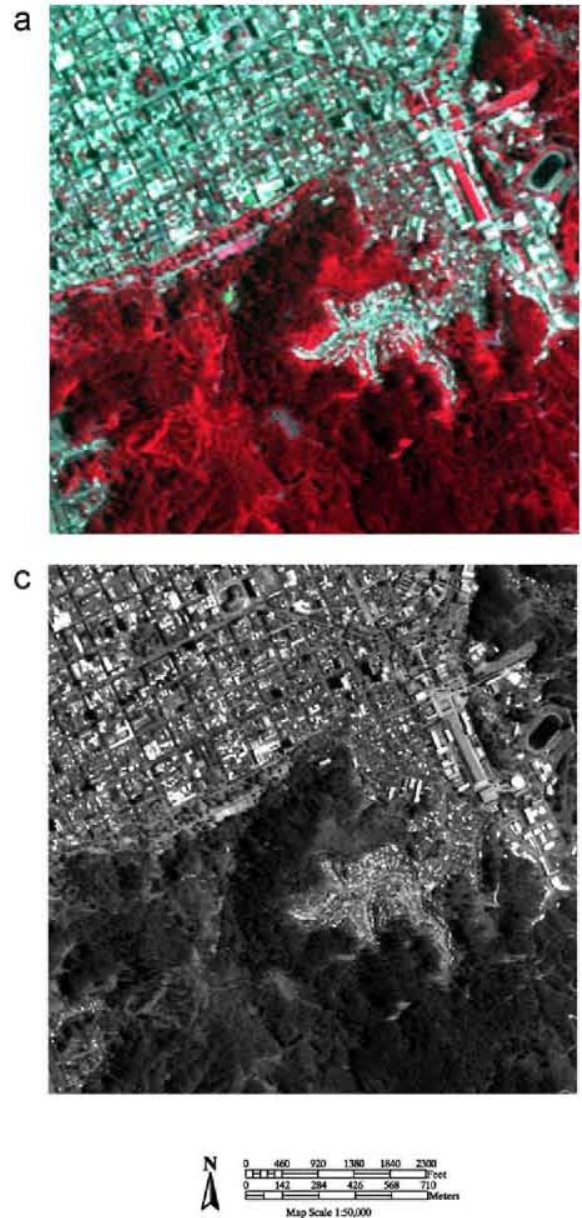

b

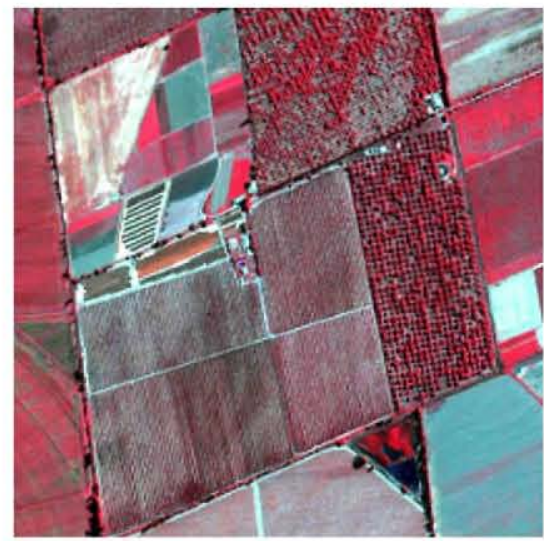

d
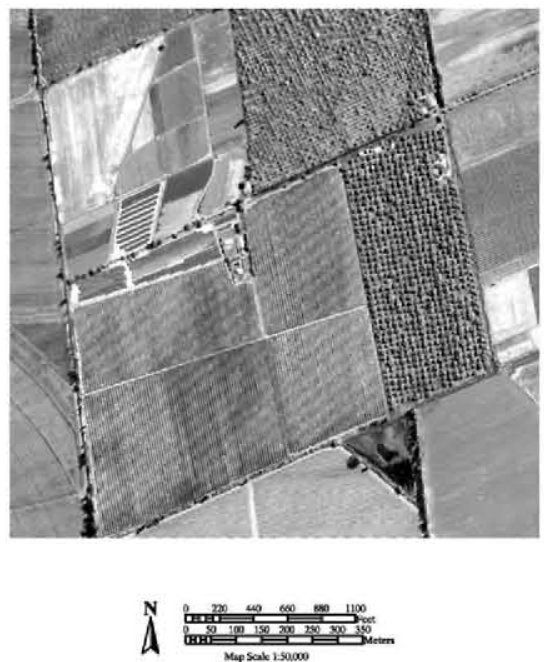

Fig. 1. Multi-spectral colour compositions (Nir-G-B) and panchromatic source images for FORMOSAT-2 (a) and (c) and IKONOS (b) and (d).

(70 33 $\left.39^{\prime \prime} \mathrm{W}, 33^{\circ} 39^{\prime} 11^{\prime \prime} \mathrm{S}\right)$ and was recorded on March 10, 2000. Both scenes have a size of $1024 \times 1024$ pixels, with a coverage area of $2.05 \times 2.05 \mathrm{~km}^{2}$ for FORMOSAT-2 and $1.05 \times 1.05 \mathrm{~km}^{2}$ for IKONOS.

\section{Results}

Before applying the proposed fusion methodology, FDMs of source images have been obtained for different values of $\omega_{\text {sizes }}$ $(7,15,31,63,127)$. Fig. 2 displays these maps for multispectralband 3 (first row) and panchromatic (second row) images of the FORMOSAT-2 scene. It can be appreciated, for multispectral as well as for panchromatic images, that increasing the $\omega_{\text {sizes }}$ value produces a smoothing effect in the characterization of the land cover roughness. On the other hand, FDMs obtained using the same $\omega_{\text {sizes }}$ value show a similar smoothing effect when the spatial resolution decreases (e.g. Fig. 2(a) $4 \mathrm{~m}$ with respect to (f) $1 \mathrm{~m}$ ).

$\alpha_{\omega_{\text {sizes }}}^{i}(x, y)$ maps have been obtained through Eq. (4). These maps have been used to obtain the fused images through the FDMF method.

From the perspective of the proposed fusion methodology, the results shown in Fig. 2 indicate that for $\omega_{\text {sizes }}=7$, the $\alpha_{\omega_{\text {sizes }}}^{i}(x, y)$ resulting will have a quasi-binary behaviour. The strong transitions between different roughness areas provide fused images with poor visual quality. At the opposite end, a value of $\omega_{\text {sizes }}=127$ gives high $\alpha_{\omega_{\text {sizes }}}^{i}(x, y)$ values, independent of the land cover type. That implies almost all information coming from panchromatic wavelet coefficients is injected into the fused image. Therefore, fused images have been obtained only for $\omega_{\text {sizes }}=15,31$ and 63 .
In this paper, the WAT fusion algorithm has been used as a reference to show that the proposed fusion paradigm reduces the artifacts introduced by the WAT fusion algorithm, in areas originally with low variability.

Colour compositions of the fused images obtained with the WAT and FDMF $\left(\omega_{\text {sizes }}=15,31\right.$ and 63$)$ methods, for the FORMOSAT -2 and IKONOS scenes are located at the top of the Figs. 3 and 4 (a)-(d), respectively. No appreciable differences in colour characteristics between images are observed when they are visually compared.

Table 3

Quality index values for FORMOSAT-2 scene.

\begin{tabular}{lllllll}
\hline Fusion strategy & $C C$ & $Z$ & SAM & ERGAS & $Q$ & SSIM \\
\hline WAT & 0.91 & 0.91 & $2.76^{\circ}$ & 1.14 & 0.41 & 0.62 \\
FDMF $\omega_{\text {sizes }}=7$ & 0.92 & 0.89 & $2.59^{\circ}$ & 1.07 & 0.57 & 0.71 \\
FDMF $\omega_{\text {sizes }}=15$ & 0.92 & 0.89 & $2.62^{\circ}$ & 1.08 & 0.56 & 0.69 \\
FDMF $\omega_{\text {sizes }}=31$ & 0.92 & 0.90 & $2.65^{\circ}$ & 1.09 & 0.54 & 0.68 \\
FDMF $\omega_{\text {sizes }}=63$ & 0.91 & 0.90 & $2.67^{\circ}$ & 1.09 & 0.51 & 0.67 \\
FDMF $\omega_{\text {sizes }}=127$ & 0.91 & 0.91 & $2.69^{\circ}$ & 1.10 & 0.48 & 0.63 \\
\hline
\end{tabular}

Table 4

Quality index values for IKONOS scene.

\begin{tabular}{lllllll}
\hline Fusion strategy & $C C$ & $Z$ & SAM & ERGAS & $Q$ & SSIM \\
\hline WAT & 0.64 & 1.00 & $2.91^{\circ}$ & 1.17 & 0.14 & 0.42 \\
FDMF $\omega_{\text {sizes }}=7$ & 0.74 & 0.94 & $2.52^{\circ}$ & 0.89 & 0.21 & 0.56 \\
FDMF $\omega_{\text {sizes }}=15$ & 0.72 & 0.97 & $2.55^{\circ}$ & 0.96 & 0.19 & 0.52 \\
FDMF $\omega_{\text {sizes }}=31$ & 0.70 & 0.98 & $2.61^{\circ}$ & 1.01 & 0.17 & 0.49 \\
FDMF $\omega_{\text {sizes }}=63$ & 0.69 & 0.99 & $2.72^{\circ}$ & 1.05 & 0.16 & 0.47 \\
FDMF $\omega_{\text {sizes }}=127$ & 0.68 & 1.00 & $2.72^{\circ}$ & 1.07 & 0.16 & 0.46 \\
\hline
\end{tabular}




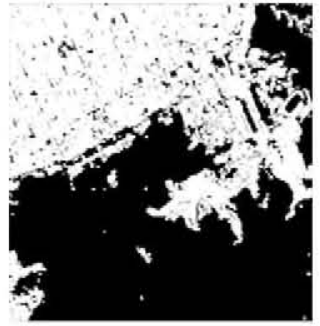

a

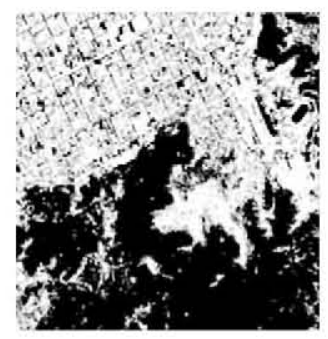

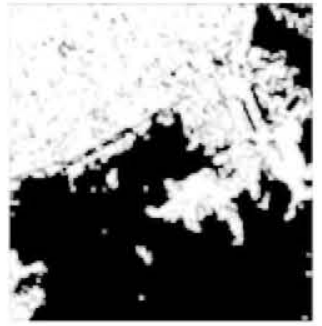

b

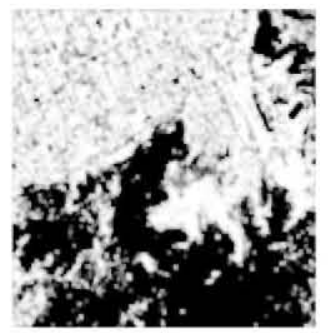

g

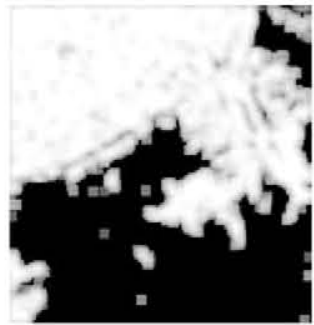

C

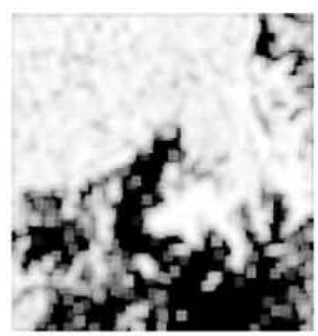

$\mathrm{h}$

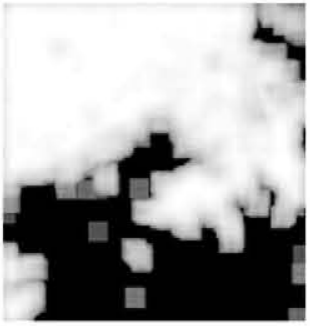

d

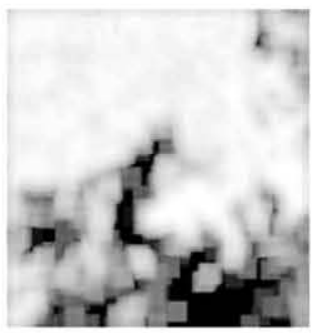

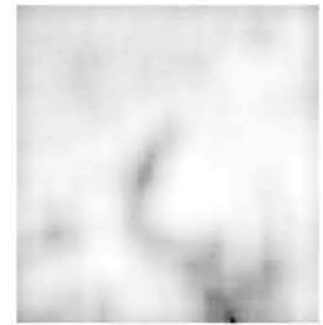

e

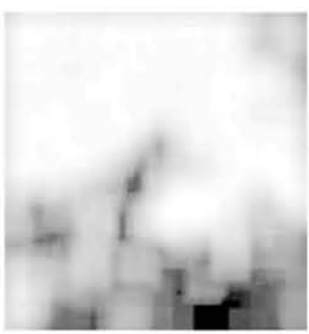

j

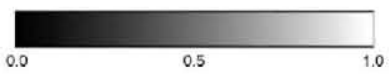

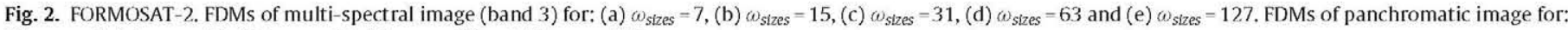
(f) $\omega_{\text {sizes }}=7,(g) \omega_{\text {sizes }}=15$, (h) $\omega_{\text {sizes }}=31$, (i) $\omega_{\text {sizes }}=63$ and (j) $\omega_{\text {sizes }}=127$.

The results of the scalar quality indices evaluation are presented in Table 3 for the FORMOSAT-2 scene, and Table 4 for the IKONOS.

According to the ideal values included in Table 1 , the analysis of the scalar indices was used to detect tendencies in fused images quality. Thus, it can observed that the spectral and global qualities decrease and the spatial quality increases as the $\omega_{\text {sizes }}$ increases. This behaviour is coherent with the FDMs behaviour (discussed above and illustrates in Fig. 2). On the other hand, when considering the scalar indices, the quality of any of the fused images obtained with the FDMF is higher than the image fused by the WAT algorithm. However, it should be noted that all scalar indices exhibit a very low sensitivity.

Q-maps obtained for fused images can be observed at the bottom of Figs. 3 and 4(e)-(h). Fig. 3(e) shows the quality differences

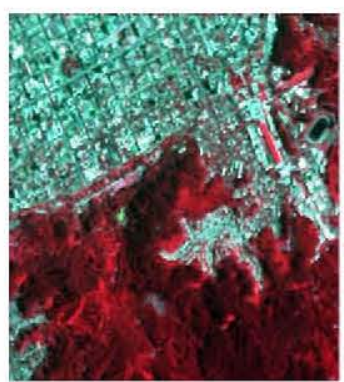

a

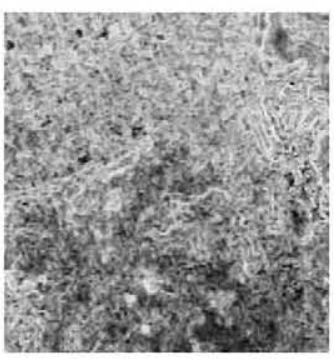

e

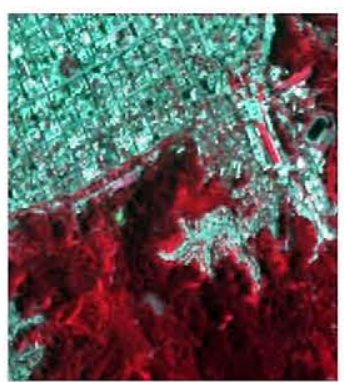

b

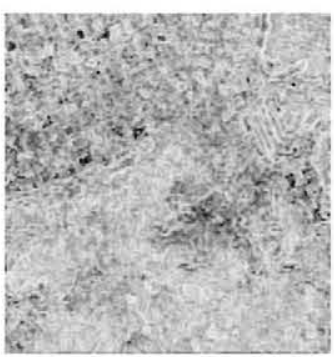

f

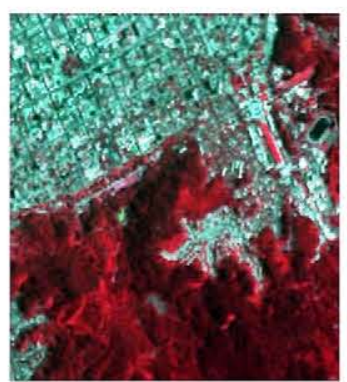

C

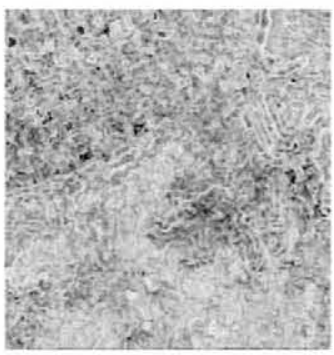

g

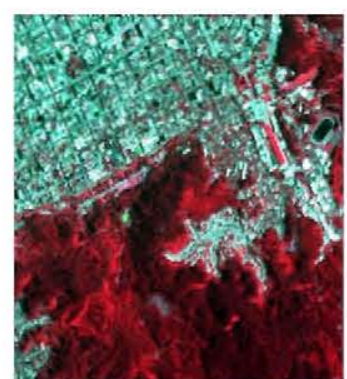

d

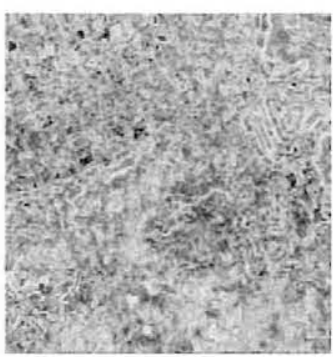

h

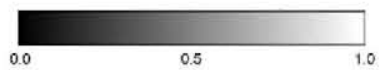

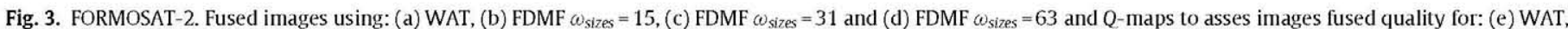
(f) FDMF $\omega_{\text {sizes }}=15$, (g) FDMF $\omega_{\text {sizes }}=31$, (h) FDMF $\omega_{\text {sizes }}=63$. 


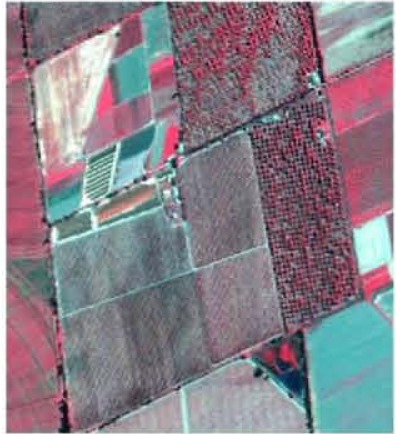

a

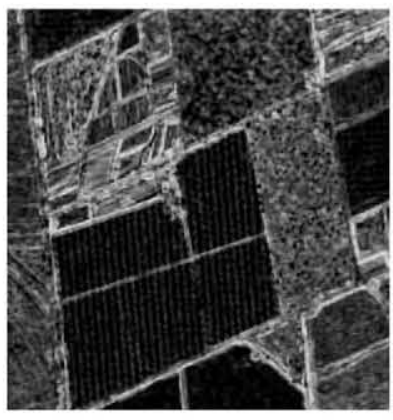

e

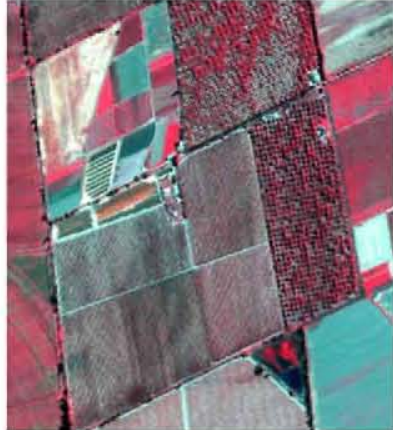

b

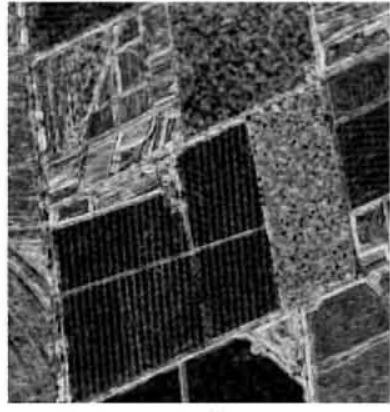

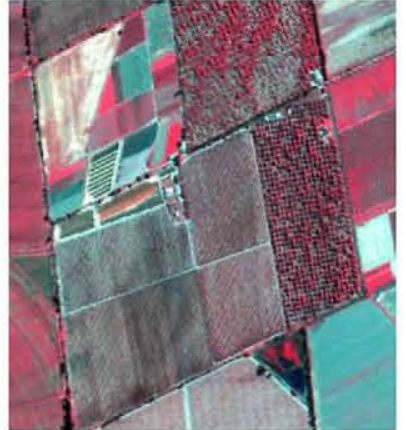

C

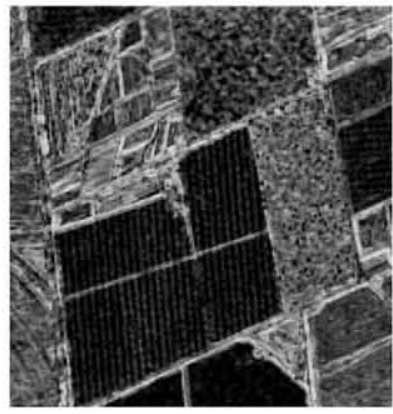

g

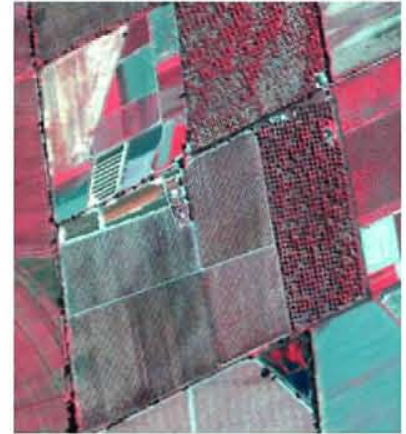

d

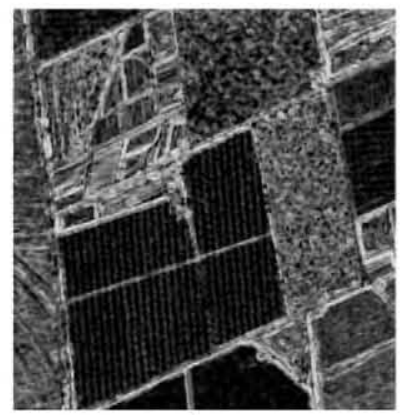

$\mathrm{h}$

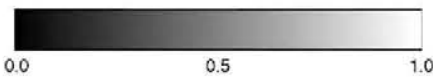

Fig. 4. IKONOS. Fused images using: (a) WAT, (b) FDMF $\omega_{\text {sizes }}=15$, (c) FDMF $\omega_{\text {sizes }}=31$ and (d) FDMF $\omega_{\text {sizes }}=63$ and $Q$-maps to asses images fused quality for: (e) WAT, (f) FDMF $\omega_{\text {sizes }}=15$, (g) FDMF $\omega_{\text {sizes }}=31$, (h) FDMF $\omega_{\text {sizes }}=63$.

Table 5

FORMOSAT-2 post-classification index (Kappa and overall accuracy).

\begin{tabular}{|c|c|c|c|c|c|c|c|c|}
\hline & \multicolumn{2}{|c|}{ Mahalanobis distance } & \multicolumn{2}{|l|}{ SAM } & \multicolumn{2}{|c|}{ Maximun likelihood } & \multicolumn{2}{|l|}{ SVM } \\
\hline & Карра & O.A. (\%) & Kappa & O.A. (\%) & Карра & O.A. $(\%)$ & Kappa & O.A. (\%) \\
\hline Original image & 0.7587 & 80.3036 & 0.7145 & 75.9821 & 0.9601 & 96.7996 & 0.9745 & 97.9702 \\
\hline WAT & 0.8327 & 86.4800 & 0.7391 & 78.1498 & 0.9477 & 95.7976 & 0.9798 & 98.3929 \\
\hline FDMF $\omega_{\text {szes }}=15$ & 0.8519 & 88.0536 & 0.8072 & 84.0298 & 0.9812 & 98.5000 & 0.9917 & 99.3393 \\
\hline FDMF $\omega_{\text {sizes }}=31$ & 0.8523 & 88.0833 & 0.8085 & 84.1369 & 0.9805 & 98.4762 & 0.9893 & 99.1488 \\
\hline FDMF $\omega_{\text {sizes }}=63$ & 0.8510 & 87.9821 & 0.8086 & 84.1488 & 0.9806 & 98.4524 & 0.9878 & 99.0238 \\
\hline
\end{tabular}

between the urban and forest coverages. This difference results because the WAT method integrates all details contained in the panchromatic wavelet coefficients in the entire fused image, independent of coverage roughness. Furthermore, there are notable differences between these Q-maps (Fig. 3(e)) and the maps shown in Fig. 3(f)-(h), where the local $Q$ values are more homogeneous for the whole image.

With respect to the fusion methodology, the IKONOS $Q$-maps (Fig. 3(e)-(h)) manifest a similar tendency as the FORMOSAT-2 Qmaps. As expected, when the spatial resolution of source images increases, the land cover variability increases. This produces lower $Q$ values for all fused images, as can be observed in Fig. 4 . Nevertheless, the results show that the proposed fusion paradigm provides higher quality fused images than those provided by the WAT method (Fig. 3).

In order to corroborate these results, fused image classification results for the FORMOSAT-2 scene were also included using four supervised classification methods: Mahalanobis distance, spectral angle mapper (SAM), maximun likelihood, support vector machine (SVM).

Ten regions of interest, with a minimum number of pixels above 1000 , were defined in the multi-spectral image. Post-classification results have been included in Table 5. From these values, it seems that FDMF method provides better post-classification indices than WAT, independent of $Q$ values.

\section{Conclusions}

A new fusion image paradigm based on fractal dimension maps (FDMs), reified through the WAT method, has been proposed. This method reduces the problem of false detail (artifacts) injected into fused images by fusion methods based on pixel-level.

It has been demonstrated that the FDMs depend on $\omega_{\text {sizes }}$ values. An increase in $\omega_{\text {sizes }}$ results in smoother transitions in the FDMs. A low value of $\omega_{\text {sizes }}<15$ implies a quasi binary behaviour of FDMs. This has a direct impact on fused image quality. Specifically, the spatial quality of the fused images increases according with the $\omega_{\text {sizes }}$, while the spectral quality has an inverse behaviour.

The Q-maps and classification results have shown that the standard WAT method does not give homogeneously quality results. In this sense, this paper shows the inability of a single value (scalar index) to estimate the quality of the entire fused image. Consequently, fused images will be better evaluated through quality 
index maps, which have the ability to show the variability of spatial and/or spectral quality.

From these results, it can be concluded that the images fused by the FDMF, as well as their classifications, present a higher quality than images fused through the standard WAT method, used as a reference in this paper.

Finally, it should be noted that the new paradigm based on FDMs can be included in any image fusion method that considers separately the low and high frequency information coming from the multi-spectral and panchromatic image, respectively.

\section{Acknowledgment}

This work is supported by Fondo de Fomento al Desarrollo Científico y Tecnológico (FONDEF). Project number: D09I1069.

\section{Appendix A.}

\section{A.1. Definition of the fractal dimension}

Natural surfaces tend to present complex characteristics that do not always adjust to a deterministic pattern or Euclidean form. Consequently, traditional Euclidean geometry is not adequate for its analysis (Parrinello and Vaughan, 2006). The Euclidean dimension of an object is related to the measurement unit used with the measured geometric value and can be obtained with (5):

$N_{L}=\left(\frac{1}{L}\right)^{D}$

where $N_{L}$ represents an object of $N_{L}$ parts scaled down by a ratio of $L$; and $D$ is an indicator relative to the complexity of the shape. It takes a value of 1 for a straight line, 2 for a surface and 3 for a volume in the case of Euclidean geometry. The generalization of this concept to fractional numbers provides the idea of fractal dimension. Thus, fractal geometry is presented in a mathematically formal framework that is capable of describing different types of complex structures with irregular forms and different scales (Mandelbrot and Maione, 1982).

The fractal dimension provides the magnitude of irregularity and/or complexity of the structure being analyzed. In the case of images (two dimensions), it is an ideal tool that can be used to determine the roughness for each type of texture (Sun et al., 2006). Different methods can be used to estimate FD. The criterion used to select the method can be based on the type of data to be processed, and on the computational complexity of algorithms (Myint, 2003; Parrinello and Vaughan, 2002).

\section{A.2. The box-counting algorithm}

One of the simplest methodologies to implement the calculation of the FD is the box-counting algorithm. This method is widely used because it offers a good trade-off between computer time and measurement exactness (Falconer, 1990). It defines the fractal dimension in the following terms: If a set $A \in R^{n}$ is covered by just-touching boxes of side with length $L=(1 / 2)^{n}$ and if the limit exists, the box-counting dimension $(D B)$ is $(6)$ :

$D B=\lim \frac{\log \left(N_{L}(A)\right)}{\log \left(\frac{1}{L}\right)}$

where $N_{L}(A)$ denotes the number of boxes of side length $L=(1 / 2)^{n}$ that intersect the set $A$ (Conci and Nunes, 2001). The $D B$ of a plane is reduced to counting the number of boxes within which the set has no null measurements. Therefore, if the limit exists, the $D B$ will be defined by (7):

$D B=\lim \frac{\log \left(N_{L}\right)}{\log \left(\frac{1}{L}\right)}$

In contrast with (6), (7) is only appropriate for square coverage rather than coverage in any form. Operatively, the algorithm consists in the consecutive partition of a binary image into cells of size $L$, where $L$ is a series of values whose minimum value would be limited by the studied image's spatial resolution. Thus, for each value of $L$, there is a number of cells $\left(N_{L}\right)$ that cover the studied object. Finally, the fractal dimension is estimated as the slope of the line that is obtained from the linear regression $\log \left(N_{L}\right)$ vs. $\log (1 / L)$.

\section{References}

Alonso, M.C., Sanz, M.A., Malpica, J.A., 2007. Classification of high resolution satel lite images using texture from the panchromatic band. In: Advances in Visual Computing, vol. 4842, pp. 499-508.

Alparone, L., Baronti, S., Garzelli, A.. Nencini, F., 2004. A global quality measurement of pan-sharpened mult ispectral imagery. IEEE Transaction on Geosciences Remote Sensing Letter 1 (4), 313-317.

Arduini, F., Fioravanti, S., Giusto, D.D., 1991. A multifractal-based approach to natural scene analysis. In: ICASSP, vol. 1-5, pp. 2681-2684.

Bruzzone, L., Carlin, L., 2006. A multilevel context-based system for classification of very high spatial resolution images. IEEE Transaction on Geosciences Remote Sensing $44(9), 2587-2600$.

Candes, E., Donoho, D., 1999. Ridgelets: A key to higher-dimensional intermittency?

Candes, E., Donoho, D., 2000. Curvelets - a surprisingly effective nonadaptive representation for objects with edges.

Chavez, P., Stuart, J., Sides, C., Anderson, J., 1991. Comparision of three different methods to merge multiresolution and multispectral data: landsat $t m$ and spot pancromatic. Photogrammetric Engineering \& Remote Sensing 57 (3) $259-303$.

Choi, M., 2006. A new intensity-hue-saturation fusion approach to image fusion with a tradeoff parameter. IEEE Transaction on Geosciences Remote Sensing 44 (6) $1672-1682$

Conci, A., Nunes, T.D., 2001. A multi-bands image analysis using local fractal dimension. In: XIV Brazilian Symposium on Computer Graphics and Image Processing, vol. 1, pp. 91-98.

Do, M., Vetterli, M., 2001. Pyramidal directional filter banks and curvelets. In: ICIP01, pp. 158-161.

Do, M., Vetterli, M., 2005. The contourlet transform: an efficient directional multiresolution image representation. IEEE Transactions on Image Processing 14 (12), 2091-2106.

Falconer, K, 1990. Fractal Geometry: Mathematical Foundations and Applications. John Wiley \& Sons, Chichester, England.

Garzelli, A., Nencini, F., Alparone, L., Aiazzi, B., Baronti, S., 2004. Pan-sharpening of multispectral images: a critical review and comparison. In: IGARSS, pp. 81-84.

Gonzalez-Audicana, M., Otazu, X., Fors, O. Seco, A, 2005. Comparison between mal lat's and the à trous discrete wavelet trans form based algorithms for the fusion of multispectral and panchromatic images. International Journal of Remote Sensing $23(6), 595-614$.

Gonzalo, C., Lillo-Saavedra, M., 2008. A directed search algorithm for setting the spectral-spatial quality trade-off of fused images by the wavelet à trous method. Canadian Journal of Remote Sensing 34 (4), 367-375.

Gonzalo, C., Lillo-Saavedra, M., Zambrano, F., Merino, F., 2008. Fusion ihs ponderada mediante dimensión fractal. In: XIII Simposio de Especialistas Latinoamericanos en Percepción Remota (SELPER).

Grazzini, J., Turie, A., Yahia, H., 2005. Presegmentation of high-resolution satellite images with a multifractal reconstruction scheme based on an entropy criterium. In: Image Processing, vol. 1, pp. 649-652.

Hallada, W., Cox, S., 1983. Image sharpening for mixes spatial and spectral resolution satellite system. In: International Symposium on Remote Sensing of Environment, pp. 1023-1032.

Huang, X., Zhang, L, Li, P., 2008. A multiscale feature fusion approach for classification of very high resolution satellite imagery based on wavelet transform. Internat ional Journal of Remote Sensing 29(20), 5923-5941.

Huiping, H., Bingfang, W., Jinlong, F, 2003. Analysis to the relationship of classification accuracy, segmentation scale, image resolution. In: IGARSS, vol. 6, pp. 3671-3673.

Karathanassi, V., Kolokusis, P., Ioannidou, S., 2007. A comparison study on fusion methods using evaluation indicators. International Journal of Remote Sensing $28(10), 2309-2341$.

Kruse, F., Lefkoff, A., Boardman, J., Heidebrecht, K., Shapiro, A., Barloon, P., Goetz, A., 1993. Interactive visualization and analysis of imaging spectrometer data. Remote Sensing of Environment 44(2), 145-163.

Li, J., Feng, Q., Guo, Q., 2008. Fractal Study of Sustainable Proportions of Natural and Artificial Oases, vol. 55. Springer, Berlin/Heidelberg, Chapter 7, pp. 1389-1396.

Li, S., Yang, B., Hu, J., 2010. Performance comparison of different multi-resolution transforms for image fusion. Information Fusion 12 (2), 74-84. 
Lillo-Saavedra, M., Gonzalo, C., 2006. Spectral or spatial quality for fused satellite imagery? A trade-off solution using the wavelet à trous algorithm. International Joumal of Remote Sensing 27 (7), 1453-1464.

Lillo-Saavedra, M., Gonzalo, C., 2007. Multispectral images fusion by a joint multidirectional and multiresolution representation. International Journal of Remote Sensing 28 (18), 4065-4079.

Mallat, S.G., 1989. A theory for multiresolution signal decomposition: the wavelet representation. IEEE Transactions on Pattern Analysis and Machine Intelligence 2 (7).

Mandelbrot, B., Maione, B., 1982. The Fractal Geometry of Nature. W.H. Freman and Company, New York.

Mitchell, H.B., 2010. Image Fusion: Theories. Techniques and Applications, 1st edition. Springer Verlag, Berlin Hidelberg.

Myint, SW, 2003. Fractal approaches in texture analysis and classification of remotely sensed data: comparisons with spatial autocorrelation techniques and simple descriptive statistics. International Journal of Remote Sensing 24 (9), $1925-1947$.

Nencini, F., Garzelli, A., Baronti, S., Alparone, L., 2007. Remote sensing image fusion using the curvelet transform. Information Fusion 8 (2), 143-156, special Issue on Image Fusion: Advances in the State of the Art.

Parrinello, T, Vaughan, RA, 2002 Multifractal analysis and feature extraction in satellite imagery. International Journal of Remote Sensing 23 (9), 1799-1825.

Parrinello, T., Vaughan, R.A., 2006. On comparing multifractal and classical features in minimum distance classification of AVHRR imagery. International Journal of Remote Sensing 27 (18), 3943-3959.

Pentland, A.P., 1984. Fractal-based description of natural scenes. IEEE Transaction Pattern Analysis and Machine Intelligence 6 (6), 661-674.

Pohl, C. Van Genderen, J. 1998. Multisensor image fusion in remote sensing: concepts, methods and applications. International Journal of Remote Sensing 19(5), $823-854$

Qu, X.-B., Yan, J.-W., Xiao, H.-Z., Zhu, Z.-Q., 2008. Image fusion algorithm based on spatial frequency-motivated pulse coupled neural networks in nonsubsampled contourlet transform domain. Acta Automatica Sinica 34 (12), 1508-1514.
Sun, W., 2006. Three new implementations of the triangular prism method for computing the fractal dimension of remote sensing images. Photogrammetric Engineering \& Remote Sensing 72 (4), 373-382.

Sun, W., Xu, G., Gong, P., Liang, S., 2006. Fractal analysis of remotely sensed images: a review of methods and applications. International Journal of Remote Sensing 27 (22), 4963-4990.

Toet, A., Hogervorst, M., Nikolov, S., Lewis, J., Dixon, T., Bull, D., Canagarajah, C., 2010. Towards cognitive image fusion. Information Fusion 11 (2), 95-113.

Tu, T., Huang, P.S., Hung, C., Chang, C., 2004. A fast intensity-hue-saturation fusion technique with spectral adjustment for IKONOS imagery. IEEE Transactions on Geoscience and Remote Sensing Letter 1 (4), 309-312.

Tu, T., Su, S., Shyu, H., Huang, P., 2001. A new look at IHS-like image fusion methods Information Fusion 2, 177-186.

Vijayaraj, V., O'Hara, C.G., Younan, N.H., 2004. Quality analysis of pansharpened images. In: IGARSS, vol. 1, pp. 85-88.

Wald, L., 2002. Data Fusion: Definitions and Architectures: Fusion of Images of Dif ferent Spatial Resolutions. Les Presses - Mines Paris.

Wang, Z., Bovik, A.C., 2002. A universal image quality index. IEEE Signal Processing Letter $9(3), 81-84$.

Wang, Z., Bovik, A.C., Sheikh, H.R., Member, S., Simoncelli, E.P., 2004. Image quality assessment: from error measurement to structural similarity. IEEE Transactions on Image Processing 13,600-612.

Yang, S., Wang, M., Jiao, L., Wu, R., Wang, Z., 2009. Image fusion based on a new contourlet packet. Information Fusion 11 (2), 78-84.

Zhang, Q., Guo, B., 2009. Multifocus image fusion using the nonsubsampled con tourlet transform. Signal Processing $89(7), 1334-1346$.

Zhou, J., Civco, D.L., Silander, J.A., 1998. A wavelet transform method to merge landsat TM and SPOT panchromatic data. International Journal of Remote Sensing 19 (March (4)), 743-757.

Zribi, M., 2010. Non-parametric and region-based image fusion with bootstrap sampling. Information Fusion 11 (2), 85-94. 